\title{
Relational Expressions in STAR File Dictionaries
}

\author{
Nick Spadaccini ${ }^{\sharp} \dagger$, Sydney R. Hall $*$ and Ian R. Castleden $\dagger^{*}$ \\ $\dagger$ Department of Computer Science and *Crystallography Centre, \\ University of Western Australia, \\ Nedlands, Perth, WA 6907, AUSTRALIA.
}

\begin{abstract}
The STAR File (Hall \& Spadaccini, J. Chem. Inf. Comput. Sci. 1994, 34, 505-508) is used widely in structural chemistry for exchanging numerical and text information with scientific journals and databases. These exchanges are increasingly dependent on data dictionaries to facilitate automatic data validation and checking. Definitions in data dictionaries are constructed using attribute descriptors and this paper describes a method attribute for specifying the relationships between data items as an executable script written in a new relational expression language called $d R E L$. The addition of this attribute improves the precision and the semantic content of dictionaries by providing relational representations of data, as well as facilitating the direct evaluation of derivable data items. The capacity to evaluate derivative data directly from the combination of primitive data and dictionary expressions is expected to change future archival approaches. The design concepts of the relational expression language $d R E L$ parser, which are applicable to any discipline, are described.
\end{abstract}

\section{RATIONALE}

The main purpose of data dictionaries is to define data items at a precision that will enable their unambiguous identification. Broadly speaking, data can be classed as either primitive or derivative. Primitive data are either experimentally measured or theoretically predicted, and need to be defined in a dictionary in sufficient detail to be unique, and so that dependencies on other experimental or theoretical parameters are well understood. Derivative data are determined from other data items, and their definition in a dictionary must specify exactly these relationships. In this paper we describe a dictionary attribute that enables the precise definition of derivative data items as relational expressions written in the script language $d R E L$. These definitions are machine interpretable and can be used to calculate derivative data directly, but can be easily specified and modified by non-experts.

We shall show that the existence of relational expressions in a text dictionary leads to data handling approaches that seamlessly integrate data values into executable definitions. These facilities are akin to those of an active knowledge base.

The rationale for direct computation from text dictionaries is many-fold. The growing complexity of computers and conventional programming languages means that scientists are increasingly removed from the detail of calculation methods, and are more dependent on "packaged" software. Current programming practices focus on computer performance and as a consequence considerable algorithmic knowledge is embedded in computer code that is difficult to understand or to document.

\footnotetext{
\# Corresponding author: nick@ cs.uwa.edu.au; phone: +618 9380 3452; facsimile: +618 93801089
} 
This practice is detrimental to both the transfer and the retention of discipline knowledge. In particular, it limits easy access to this knowledge, and therefore to its extension and application. The problem is not diminished with recent computing developments. Modern languages and implementation tasks increasingly require formal training in computer science or software engineering. Certainly the era when major scientific packages were written by discipline experts for ready modification by other scientists has passed and there is an real need to develop approaches that can provide simpler links between discipline knowledge and computational outcomes.

Data dictionaries containing relational expressions are one such approach. Used in conjunction with databases they provide electronic information that is extensible, interactive and accessible. They are of enormous value as a pedagogical resource. For example, scientists seeking specific advice and information within their discipline often use Internet news-groups. Such an approach is not as reliable or extensive as accessing interactive data dictionaries that have been constructed by discipline experts.

In this paper we describe a dictionary relational expression language $d R E L$ that is functional and intuitive for humans, and amenable to machine interpretation. It is sufficiently symbolic to be easily translated into textbook information. It builds upon the previously reported dictionary definition languages $[7,8]$ for the CIF dictionaries which form part of the STAR File exchange approach $[1,2]$ widely adopted by the chemical structure community. The prototype version of the dictionary used as a proof-of-concept for the $d R E L$ relational expressions has been defined using structural science data, but exactly the same principles apply to data in other fields.

We shall also show that data dictionaries are simple text files that are made executable by translation and then compilation into Java byte-code. This compiled dictionary contains routines for parsing STAR data files. Data evaluation using the compiled dictionary is not fast by normal computational standards, but it is certain to benefit from future advances in computer technology. More to the point, we maintain that the most important measure of "efficiency" is the speed with which users can specify new relational expressions, and understand what has been previously defined.

\section{BACKGROUND}

A STAR File format $[1,2]$ used in the dictionaries described in this paper, is a universal file structure for exchanging and archiving data electronically. A discipline-specific application of this approach, the crystallographic information file[3] (CIF), is used widely in structural chemistry for journal and database purposes[4,5]. The STAR File and CIF were developed in the late 1980's in response to the rapid growth in measured and calculated scientific data, and the need to interchange information electronically over the increasingly accessible Internet. The adoption of the CIF in 1990 by the International Union of Crystallography, as the recommended standard for the exchange of data, led to its extensive application to structural science for data submission to both journals and databases.

The development history of data dictionaries in structural science is relevant here as it illustrates the importance that some scientific disciplines are attributing to the careful organization and delivery of knowledge, largely in response to massive increases in data. In structural sciences the CIF development has, in addition to promoting open and flexible information exchange, stimulated the compilation of data dictionaries which have been internationally approved by the governing bodies in a number of sub-disciplines[6]. CIF and STAR files use unique tags to identify exchanged data items and these tags are defined in dictionaries written in a dictionary definition language[7,8] 
(DDL). Dictionary definitions are composed of attributes that ascribe particular properties to the item e.g. its data type, its permitted values, and so on. These attributes represent the vocabulary of a STAR DDL, and determine the richness with which data items can be defined.

Data dictionaries support CIF exchange and archival processes by uniquely identifying and classifying data items. They also play a pivotal role in the automatic validation of exchanged data. Currently two dictionary languages are used in structural chemistry, DDL1.4[7] for core crystallographic and powder diffraction items[6] and DDL2.1[8] for the macromolecular structure items. Both DDL versions involve similar attributes; however, DDL2.1 contains a richer attribute set. The access and validation of data using dictionaries is strongly dependent on the scope and precision of the DDL attributes. The developments described in this paper have highlighted a need for at least three major additions to the DDL attributes (1) stronger "typing" of data i.e. the inclusion of "container" types such as lists, matrices, vectors, etc., (2) allow for the hierarchical classification of data and for inheritance, and (3) an intuitive expression language that permits the concise representation of algorithmic relationships between data items.

In this paper we will mainly describe the purpose and properties of the DDL method attribute written in $d R E L$. The detailed specification of the $d R E L$ syntax will be the subject of later paper.

\section{THE Role OF THE METHOd ATtRIBUTE}

Method attributes serve to propagate the values of primitive data items into derivative items. As discussed earlier, primitive data result from measurement or theory, and are irreducible. Derivative items can be calculated from other data by following a known algorithm. This derivation is normally achieved using customized computer software. We propose an alternative approach in which the method attribute of a derivative item provides this algorithm in the form of a machine-interpretable text expression.

Method attributes serve two important purposes. They contain data relationships that constitute precise usable knowledge, and they provide executable algorithms that may be used to evaluate the defined item in terms of others. Such attributes provide a simple, transparent and direct approach to automating and generalizing processes which are currently either ad hoc, or, at best, highly customized. Of longer-term importance is that precise relational information in data definitions is an essential requirement in extending STAR dictionaries into the realm of active knowledge bases.

A number of major and immediate benefits arise from this approach.

\section{- Enhancement of knowledge}

The use of analytical expressions in data definitions has the obvious pedagogical benefit of making this information more readily accessible to humans. Relational expressions written in $d R E L$ represent symbolic, self-descriptive and mathematically logical descriptions that are easy to read and to understand. The underpinning objectives of the $d R E L$ language are to simplify this information for all dictionary users, and yet be sufficiently powerful to attract application experts. Furthermore, the method attribute, along with all other dictionary attributes, is amenable to the creation of a "textbook" representation of the definitions. A prototype parser is already available that can create a PDF document from existing CIF dictionaries[9]. It is anticipated that future CIF definitions will be rendered automatically into a mathematical typeset (using TEX or MathML), so that pedagogical documents of defined knowledge can be produced directly from a DDL dictionary. 


\section{- Retention of knowledge}

The loss of discipline-specific technical knowledge, such as computing algorithms, is an increasing problem with the evolution of computer technology and programming languages. The erosion occurs in many ways; when computer software is unsupported by a new generation of hardware; when computing languages evolve and earlier versions are incompatible; when program authors are no longer accessible; or simply in those cases when knowledge is obscured by the programming language. The dictionary methods proposed herein offer a long-term solution because they promote the retention of computer algorithms in terms of a minimal-style language that enables relationships to closely resemble mathematical formulae. The dictionary method attribute contains the algorithms as transparent, machine-independent, yet executable expressions.

\section{- Reduction of data archives}

A large proportion of data currently exchanged and archived is superfluous, in that they can be easily derived from primitive data if the interdependencies are known. The use of dictionary methods to directly evaluate derivative items will promote new and yet-unthought-of data handling paradigms. It will reduce the need to exchange or archive derivative data that can be generated from dictionary methods. Additionally, these methods can be used to automatically verify and validate existing derivative data values. For example, this approach can replace customized software performing validation tasks for scientific publishers[10].

\section{- Evaluation of data}

The most important long-term advantage of the $d R E L$ methods is computational rather than pedagogical. Executable expressions in $d R E L$ avoid most of the obfuscation problems of existing imperative coding techniques and offer a programming paradigm empowering scientists to control and modify their computations in ways paralleled only by symbolic programming approaches within existing algebraic computing packages.

\section{AN EXAMPLE OF THE METHOD ATTRIBUTE}

Within the DDL formalism, each attribute is assigned a unique name or tag. A variety of attribute specifications are illustrated in three simple definitions of data items representing the cell characteristics of a crystal, as shown in CHART 1. For example, the attributes_type.container and _type.value specify that the data TYPE for the data item_crystal.density (in CHART 1a) as a SINGLE, REAL number. Each attribute in these definitions serves to identify a particular property of the defined item.

\section{$\ll<<<<$ CHART 1}

The attribute_method.expression contains an expression for evaluating the defined item in terms of other defined items. The way that this is applied is best illustrated using a simple example. The data dictionary definition of the crystal density shown in CHART 1a has a method attribute _method.expression containing the simple $d R E L$ expression

$$
\text { _crystal.density <- } 1.6605 \text { *_cell.mass / _cell.volume }
$$

This expression defines the density $\boldsymbol{d}$ of a crystalline solid (in $\mathrm{Mg} / \mathrm{m}^{3}$ ) as the ratio of the atomic mass within a crystal unit cell and the volume of that cell, with the mass in units of daltons and the volume in cubic angstroms. The _method.expression attribute enables the crystal density to be 
evaluated if both the mass and volume values are known. Otherwise,_cell .mass and _cell.volume are treated as functions[13] which need to be evaluated from their dictionary definitions, which are included as CHART $1 \mathrm{~b}$ and $1 \mathrm{c}$, respectively. This reduction approach to evaluation continues until either the density value is determined, or all derivation pathways are exhausted. It is important to note that the relational expressions are kept as simple and as modular as possible. Second-order relationships, such as those needed to derive_cell.mass or _cell.volume are not considered part of the_crystal.density method.

\section{$\ll<<<$ CHART 2}

CHART 2 shows a small test data file in which the item_crystal.density is flagged as unknown with a "?". Parsing this file using $d R E L$-compiled definitions shown in CHART 1 produces an output file shown in CHART 3. This contains a value of _crystal.density calculated from the method expressions, along with an appended standard uncertainty value derived from these expressions. Note that there are a number of other data values determined as a consequence of the derivation processes (shown in bold font).

\section{$\ll<\ll<<$ CHART 3}

One further lesson can be drawn from these simple example definitions. Note that the relational expression in the method of CHART 1a can be easily parsed to derive the formula,

$$
\text { density }_{\text {crystal }}=\frac{1.6605 \text { mass }_{\text {cell }}}{\text { volume }_{\text {cell }}}
$$

and is very similar to the textbook definition of the crystal density,

$$
\boldsymbol{d}=\frac{1.6605 \boldsymbol{M}_{c}}{\boldsymbol{V}_{\boldsymbol{c}}}
$$

Similarly, the expression for the volume of a crystal cell shown in the method of CHART $1 \mathrm{~b}$,

$$
\text { volume }_{\text {cell }}=\boldsymbol{a}_{\text {cell_vector }} \cdot\left(\boldsymbol{b}_{\text {cell_vector }} \mathbf{x} \boldsymbol{c}_{\text {cell_vector }}\right)
$$

is explicit because vector quantities can be used in the dictionary definitions. Although the expression for crystal cell mass in the method of CHART 1c,

$$
\text { mass }_{\text {cell }}=\sum_{\mathrm{t}}^{\text {atomic_type }}\left(\text { number_in_cell }{ }_{\mathrm{t}} * \text { mass }_{\mathrm{t}}\right)
$$

is not as straightforward as for the density and volume, the syntax involving a Loop of products in the at om_type list matches the structure of the data file (see CHART 3). The in-place operator "+<-" in the method script signals a summation of these terms.

These introductory examples illustrate that dictionary attributes provide for more than just derivative evaluation. They restrict data quantities in terms of typing, enumeration (e.g. all values must be nonnegative real numbers) and expected units. These are used to validate existing data values.

\section{THE REDUCTION APPROACH}

As the crystal density definition has shown (see CHART 1), it is operationally advantageous to treat data items in $d R E L$ relational expressions as functions, and to employ a reduction approach to their evaluation. This simplifies the compilation and maintenance of dictionary definitions by enabling new method scripts to be added with little or no knowledge of data dependencies in the existing 
definitions. As a consequence, method scripts need only contain the most directly related items (e.g. in the density method these are the cell mass and volume items, not the cell vector items, etc.), with no prior knowledge of how these are to be evaluated. This type of modularity promotes simple, concise and symbolic relational expressions.

Instead of placing the highest priority on execution efficiency, the $d R E L$ approach emphasises the clarity of data relationships. This has important implications for the long-term retention of knowledge because algorithms are no longer obscured by the syntax of conventional programming languages. The philosophy underpinning the development of the dictionary relational expression language $d R E L$ is that knowledge be retained in its most comprehensible human-readable form, independent of current computational constraints. Because large improvements in computer performance are certain, human, rather than machine, efficiency has been made the main consideration.

The relative importance of expressing knowledge intuitively needs some elaboration. Most computer software is written in imperative procedural code that is largely incomprehensible to nonprogrammers. To illustrate this we contrast the expression used the volume definition in CHART $1 \mathrm{~b}$,

$$
\mathbf{V}=\boldsymbol{a} \cdot(\boldsymbol{b} \mathbf{x} \boldsymbol{c})
$$

where the volume $\mathbf{V}$ of a parallelepiped is expressed in terms of its cell vectors $\boldsymbol{a}, \boldsymbol{b}$ and $\boldsymbol{c}$, with the conventional approach to coding a volume calculation in which the vectors are expressed in terms of the scalar cell lengths $a, b$ and $c$ and angles $\alpha, \beta$ and $\gamma$, as

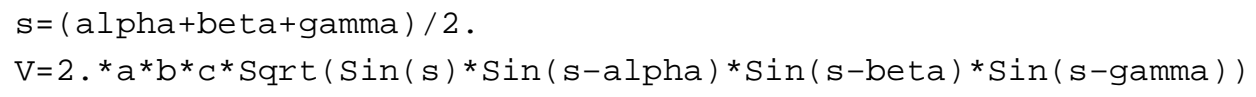

This expanded code may be computationally efficient, but its intent is obscure, compared to the dREL expression shown in CHART $1 \mathrm{~b}$. The usual coding practices used in $C, C++$ and Fortran languages also complicate the algebraic structure of relationships, and this limits their use for noncomputational purposes, such as translation through algebraic manipulation software. 


\title{
DESIGN CONCEPTS OF $d R E L$
}

\begin{abstract}
"Conventional programming languages are growing evermore enormous, but not stronger. Inherent defects at the most basic level cause them to be both fat and weak: the primitive word-at-a-time style of programming inherited from their close ancestor - the von Neumann computer, their close coupling of semantics to state transitions, their division of programming into a world of expressions and a world of statements, their inability to effectively use powerful combining forms for building new programs from existing ones, and their lack of useful mathematical properties for reasoning about programs." Backus, 1978.
\end{abstract}

Backus [11] expressed these widely shared concerns in his acceptance speech for the 1977 ACM Turing Award. Imperative programming languages are efficient because they mimic the von Neumann architecture, but in doing so they obscure the meaning of the computed algorithm. Despite this serious limitation, it has only been with the most recent improvements to computer performance that serious consideration has been given to alternative programming rationales, such as provided by functional languages. These have been successful with some numerical computing tasks[12,13], but have been little used in scientific applications.

In the design of $d R E L$, the benefits of the functional, procedural and object-oriented programming paradigms have been considered. As emphasised earlier, we gave the execution efficiency of method scripts a lower priority to other $d R E L$ objectives, such as expression simplicity, conciseness and clarity. It was rationalized that future improvements to computer performance would improve execution efficiency, but only a better language design would lead to similar efficiency gains for the average user. These considerations made the prospect of designing an intuitive language with good operational properties an achievable goal.

\section{dREL Design Criteria}

The principal design criteria for $d R E L$ were the following.

1. The relational expressions in a DDL method attribute of a dictionary definition must be linked operationally to all other DDL attributes within that definition, and, to other related definitions in the dictionary. The language scope must encompass the entire contents of a dictionary in order that all data items and their attributes are accessible in the execution of a method script.

2. The execution of a method script can only produce a result if the values of dependent data items can be determined. Ultimately, each evaluation depends on the availability of one or more data values from the data file being parsed by the dictionary application. It follows that the $d R E L$ parser must be completely conversant with the STAR File syntax and structure, and must have access to particular known values in a data file in order that evaluation can occur.

3. Relational expressions should mimic corresponding mathematical formula as closely as possible. A comprehensive set of data "container" types, e.g. Complex, List, Tuple, Table, Vector and Matrix must be supported by $d R E L$ and be manipulated without explicit iteration over the elements of the container type. Operator overloading based on operand types should be supported. An example of this is given in the penultimate statement of the code fragment in CHART 6 in which the argument $\boldsymbol{h}^{*} \mathbf{S} * \boldsymbol{x}$ represents the multiplication of a matrix $\mathbf{S}$, by the row vector $\boldsymbol{h}$ and the column vector $\boldsymbol{x}$ ). 
4. dREL must support a data type referred to as Measurement. These are data which are not exact i.e. each number has an associated uncertainty value (e.g. the measurement value recorded as $10.42(6)$ means there is a .667 probability that the true value is in the range $10.36-10.48)$. The statistical uncertainties of measurement data in a relational expression must be handled automatically. That is, the evaluation of a method script involving any data of type Measurement must result in a value of this type and have an associated uncertainty value. Mathematically, the expression for calculating this uncertainty is function of the partial derivatives of the original evaluation expression, and this can be generated using standard computer algebraic approaches. Accordingly, an expression language must promote the use of symbolic representations that can be differentiated algebraically.

\section{Dictionary Attribute Requirements}

The $d R E L$ design is dependent on the versatility and scope of the dictionary DDL syntax and attributes[7,8]. The most important of these considerations are as follows.

1. Each definition in a dictionary should be an encapsulation of the properties of a specific data item, and that this entry be treated as a discrete object. During execution these dictionary objects are married to specific data values from a data file.

2. That data items be classified into groups of related items, referred to in a STAR File as a category, and that this classification is declared explicitly in the dictionary. In this context a data item may be considered an attribute of the category. For example, the data items _atom_site.label and_atom_site.fract_xyz (see CHART 4 and 5) are members of the ATOM_SITE category. Within this category they may be accessed through their data name extensions, label and fract_xyz.

3. A single data item may have a list of values. A category of such items may be thought of as a table in which the columns contain the values and the item data names form the column header. A category of list items must have at least one data item with unique values. This item is the category key for uniquely referencing a loop packet (i.e. a row in the table).

\section{$\ll<<<<<$ CHART 4}

4. Data categories may be divided into three classes according to their roles. They are major function, hierarchical and derivation. Identifying these roles assists in the correct definition of data classes, and in how the method attributes will be parsed by $d R E L$. This is best conceptualized as a three-dimensional organizational model. The major function categories each occupy one axis of organizational space. In structural sciences these would be the major data classes CRYSTAL, DIFFRACTION, STRUCTURE and MODEL, representing the families of data separated by purpose, origin or computational progression.

A second dimension of the organizational model separates hierarchical categories. Such categories form families of data that are potentially merged into a common parent category. For example,ATOM_SITE data is part of the STRUCTURE family, and ATOM_SITE_ANISO is a child member of the ATOM_SITE category. The knowledge of such relationships is important because $d R E L$ merges child categories containing list data into the lists of parent categories of the same family (see CHARTS 4 and 5).

The third organizational dimension separates derivation categories. These categories contain data that are derived from other data. They are separated in this dimension by the order in which they 
can be derived: primary, secondary, tertiary, quaternary, such as with ATOM_SITE, MODEL_SITE, GEOMETRY and ENTITY.

\section{$\ll<<<<<$ CHART 5}

5. Data items in list categories related by hierarchy may be merged with "parent" categories in the same family because their category key items are equivalent and interchangeable. CHART 4 illustrates such a relationship for ATOM_SITE data, and CHART 5 shows how data in these list categories may be operationally "joined".

6. Data items belonging to list categories related by derivation share common category keys. These keys are explicitly specified in the dictionary so that lists related by derivation can be mapped onto each other.

\section{dREL Operational Requirements}

Operational requirements of $d R E L$ arise specifically from the need for assignments in the method scripts. These have had a significant bearing on the $d R E L$ design criteria.

1. Defined data names (i.e. tags) appearing on the right hand side of relational expressions are treated by $d R E L$ as functions, in that they reference a specific value, or they refer to a method script in the dictionary that can be evaluated.

2. The evaluation of specific data items in a relational expression take place on an "as needed" basis, just as in functional languages.

3. dREL statements involving an imperative syntax will be supported to control access of stored variables in certain cases.

\section{dREL RUN-TIME SCOPING}

Although the specification of the scoping rules for $d R E L$ relational expressions is the topic of a later paper, we give a summary of these requirements here to help explain the fundamental differences between $d R E L$ and other scripting languages. The run-time evaluation model employed by $d R E L$ has similarities to existing functional languages, but differs markedly in that it is based on the specific scoping requirements for accessing STAR File data. They are as follows.

1. A STAR data tag in a $d R E L$ script represents a pointer to either its value in an associated STAR data file, or its method script from which a value can be derived. Once evaluated, the pointer is replaced by the value. In this way, evaluation occurs only once.

2. An evaluation in a method script is postponed if the process branches to another method script, and the new script assumes the same scope and context for data items from the previous process.

3. Only the data item within the current scope is evaluated. In the case of repeated (list) data items, only one loop packet of data is, by default, in scope. Examples of this scoping requirement are illustrated in CHART 6.

4. For list categories, the local-reference pointer to the current loop packet (represented by the current value of the defined category key) in the statement

With local-reference as category-name 
Data items in the category category-name are addressed by appending their data name extension to the identifier local-reference. Hence, the statement,

$$
\text { With a as atom_site }
$$

places the items in the current packet of the ATOM_SITE category in scope, and these can be accessed as, say, a.label, a.fract_xyz, etc. The scope of the "With" statement is unchanged from the prior evaluation sequence. For example, if the joined list shown in CHART $5 \mathrm{~b}$ is being processed, and the current loop packet in scope has a category key value of _atom_site.label $=02$, then the invocation of a new method containing the statement

With s as atom_site

will not change the scope of a.

5. For list categories, packets of data are successively brought into scope by the statement

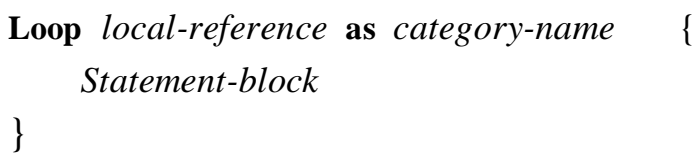

For the example in 5. above, the statement

$$
\text { Loop a as atom_site \{ \} }
$$

implies that pointer to all loop packets (identified in CHART $5 b$ as the category key items _atom_site. label with the values o1 to o3) will be successively placed in scope.

\section{FURTHER dREL APPLICATIONS}

Some examples of complex evaluation methods are now needed to illustrate the full versatility of the $d R E L$ approach. The first example calculates the structure factor value for specific diffraction vectors and this shows, in particular, the operation of the reduction process during data evaluation. The second example method determines the list of inter-atomic angles within a molecular structure and this illustrates how ab initio lists of items are created from more primitive data in the derivation pathway.

\section{EXAMPLE 1. A crystallographic structure factor calculation}

For crystallographic studies, the determination of molecular structure from diffraction data requires the measured structure factors be compared with those values calculated from the proposed molecular model of individual atom sites. The calculated structure factor $F(\boldsymbol{h})$ for each diffraction point, $\boldsymbol{h}=h, k, l$, 


$$
F(\boldsymbol{h})=\sum_{\mathrm{j}=1, \mathrm{~N}} \mathbf{f}_{\mathrm{j}}(\mathrm{s}) \mathrm{e}^{-\mathbf{B}_{\mathrm{j}}(\mathrm{s})} \mathrm{e}^{-2 \pi \mathrm{i} \boldsymbol{h} . \boldsymbol{r}_{\mathrm{j}}}
$$

is a Fourier transform expressed as the summation of the $\mathrm{N}$ atom sites in the unit cell, where $\mathrm{fj}$ is the atomic scattering as function of the scattering direction $\mathrm{s}, \mathrm{e}^{-\mathbf{B}_{\mathrm{j}}(\mathrm{s})}$ is the atomic displacement factor and is a function of the scattering direction $\mathrm{s}$, and the atomic site coordinate vectors $\mathrm{r}_{\mathrm{j}}\left(=x_{\mathrm{j}} / a, y_{j} / b\right.$, $\left.z_{j} / c\right)$. When the crystal unit cell contains atomic sites related by space group symmetry, and the expression for the structure factor becomes

$$
F(\boldsymbol{h})=\sum_{\mathrm{j}=1, \mathrm{U}} \mathbf{f}_{\mathrm{j}}(\mathrm{s}) \sum_{\mathrm{k}=1, \mathrm{M}} \mathrm{e}^{-\mathbf{B}_{\mathrm{j}}(\mathrm{s})} \mathrm{e}^{-2 \pi \mathrm{i} \boldsymbol{h} \cdot \boldsymbol{S}_{\mathrm{k}} \boldsymbol{r}_{\mathrm{j}}}
$$

where $S_{\mathrm{k}}$ is the Seitz matrix for the $\mathrm{k}^{\text {th }}$ symmetry transformation, and the summation is partitioned into the number of unique atomic sites $U$ and the number of symmetry matrices $M(\mathrm{~N}=\mathrm{UxM})$. The dictionary definition for a calculated structure factor is shown in CHART 6 and the attribute _method.expression contains a relational expression representing the above equation.

\section{>>>>>>>>> CHART 6}

The different attributes in a dictionary definition contribute to the evaluation of _refln.F_complex, which is a single complex number belonging to the REFLN category. Its evaluation from_method.expression is dependent on the values of other data items, _atom_site.fract_xyz, _atom_site.type_symbol, etc., and these must be available from either an associated data file or through the method scripts in their definitions. The evaluation of the calculated structure factor involves the following basic steps. These steps are typical of all $d R E L$ evaluation processes.

Step 1. A STAR file containing list data in the category REFLN is parsed sequentially. Each loop packet can be uniquely identified by the value of its category key_refln.hkl. The evaluation of each_refln.F_complex in the REFLN list may be requested simply by the presence of a "?" in place of a value. The evaluation of _refln. F_complex is made for each loop packet independently.

Step 2. If the value_refln.F_complex for a specific_refln.hkl value is available from a prior evaluation, it will be accessed directly, and the _refln. F_complex method script is not processed.

Step 3. If the value of_refln.F_complex is unknown for the specific_refln.hkl value, the parser processes the _method.expression script inserting the values of the referenced data items from either the associated STAR data file, or from processing the defined method scripts of these referenced data items.

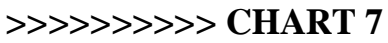

A typical derivation pathway for Step 3 in this calculation is shown in CHART 7. Method $A$ is the _method.expression script for_refln.F_complex. Methods $B$ and $C$ are applied only if the values of _refln.form_factor_list and_function.adp_factor are not available from the data file or from a prior evaluation. The arrows in CHART 7 represent the branches to the methods of the items, _refln.form_factor_list and_function.adp_factor. The parser continues to "stack" incomplete evaluations until all the needed items have been determined, or the possible derivation pathways have been exhausted. 


\section{EXAMPLE 2. Creating $a b$ initio list data}

This example is intended to show how lists of derivative data are generated $a b$ initio from the dictionary definitions in the event that a requested data item is not present in a data file. In previous examples the data name of the required data item was present in the data file, but with a missing value i.e. "?". When there are no tags in the data file for a requested category of data, the _method.expression within the category definition is used to construct a list of data items for this category. This example also shows how the calculation sequence flows automatically to other categories referred to in the method expression. In this instance the categories form a derivation pathway that link the atom coordinate data (available from the data file) to the methods that evaluate the angles between bonded atomic sites in a molecule. The derivative pathway is as follows.

The list of atomic coordinate data (category ATOM_SITE) are present in the data file. $\downarrow$

The list of "connected" atom sites in a molecule (category MODEL_SITE) are calculated. $\downarrow$

The list of molecular angles (category GEOM_ANGLE) are calculated.

Note that this sequence of calculations requires the generation of an intermediate list of connected sites; a step that arises automatically from the relationships of the dictionary definitions.

With the request for molecular angles, the $d R E L$ evaluation process proceeds as follows.

Step 1. The request for either_geom_angle.value or the GEOM_ANGLE list cannot be satisfied from the data items present in the STAR data file shown in CHART 2. This file contains the crystallographic results for the hypothetical molecular structure shown.

Step 2. The parser then refers to the attribute_method.expression in the GEOM_ANGLE category definition, shown in CHART 8, for the expressions needed to calculate the inter-atomic angles from the MODEL_SITE list of connected atom sites. However, these sites are also not available from the STAR file.

Step 3. The parser then refers to the attribute_method.expression in the MODEL_SITE category definition for the expressions needed to calculate the connected atoms sites in the molecule from the ATOM_SITE list of symmetry-unique atom sites.

Step 4. The ATOM_SITE list of sites are present on the STAR file (see CHART 2), and the parser proceeds to generate the connected atom sites based on the algorithm in the MODEL_SITE category definition. This results in the MODEL_SITE list shown in CHART 9a.

Step 5. The parser process stack now reverts to the algorithm in the GEOM_ANGLE category definition. This shows that if the positions of three connected atoms in the MODEL_SITE list are known in terms of the vectors $\boldsymbol{r}_{1}, \boldsymbol{r}_{2}, \boldsymbol{r}_{3}$ in a Cartesian system then the angle $\phi$ subtended at site 2 is

$$
\operatorname{Cos} \phi=v_{21} \cdot v_{23} / \mathrm{d}_{21} \mathrm{~d}_{23}
$$

where $\boldsymbol{v}_{21}=\boldsymbol{r}_{\boldsymbol{1}}-\boldsymbol{r}_{2} ; \boldsymbol{v}_{23}=\boldsymbol{r}_{3}-\boldsymbol{r}_{2}$ are the inter-atomic vectors and $\mathrm{d}_{21}=\left|\boldsymbol{v}_{21}\right| ; \mathrm{d}_{23}=\left|\boldsymbol{v}_{23}\right|$ are the interatomic distances. This step produces the GEOM_ANGLE list shown in CHART 9b. 


\section{CONCLUSION}

We have shown that the addition of relational expressions as method attributes of STAR data dictionaries can promote their use as active knowledge bases. The rationale and the purpose of these attributes are described. These provide for much greater clarity and precision in the definition of data. The availability of method scripts for use in future discipline-specific dictionaries, and in laboratory dictionaries containing local information, will promote the scientific exchange and exploitation of data, well beyond that currently achievable.

Relational expressions in $d R E L$ are simple, concise and algorithmic, and this greatly improves the human readability and interpretation of definition information. Additionally, because method attributes are executable, derivative data can be directly evaluated as needed. This means that much derivative data need not be stored in data files, reducing the volume of data exchanged and archived.

Access to executable method scripts in dictionaries offer the promise of new computing paradigms for future scientific applications. Calculations can be initiated simply be requesting the value of a specific data item. The calculation sequence and algorithms will be determined entirely by the method attributes encountered in the evaluation pathway.

A prototype parser for $d R E L$ may be viewed at the web site www.cs.uwa.edu.au/dREL. The executable versions of the examples presented in the paper are available at that address, as is information on future versions of $d R E L$, and related publications. The source code of $d R E L$ will in the future be distributed as open system software entitling users to free use and code access, conditional that new developments can correctly parse supplied compliance scripts.

\section{REFERENCES AND NOTES}

1 Hall, S.R. The STAR File: A New Format for Electronic Data Transfer and Archiving. J. Chem. Inform. Comp. Sci. 1991, 31, 326-333.

2 Hall, S.R.; Spadaccini, N. The STAR File: Detailed Specifications. J. Chem. Inform. Comp. Sci. 1994, 34, 505-508.

3 Hall, S.R.; Allen, F.H.; Brown, I.D. The Crystallographic Information File (CIF): A New Standard Archive File for Crystallography. Acta Cryst. 1991, A47, 655-685.

4 Electronic submission of material in the CIF format is recommended by

American Chemical Society (pubs.acs.org:80/supmat/suppdoc.html)

Royal Society of Chemistry (London) (www.rsc.org/is/journals/authrefs/submit.htm)

International Union of Crystallography (www.iucr.org/iucr-top/ciffindex.html)

CSIRO/Australian Academy of Science (www.publish.csiro.au/journals/ajc/)

Oldenbourg Verlage (www.oldenbourg.de/verlag/zkristallogr/)

5 Submission of data in the CIF format is requested by

Nucleic Acid Database, Rutgers University (ndbsever.rutgers.edu)

Protein Data Bank, Rutgers University (www.rcsb.org/pdb)

Cambridge Structural Database (www.ccdc.cam.ac.uk)

Powder Diffraction Database (www.icdd.com)

Inorganic Crystal Structure Database (www.fiz-karlsruhe.de/stn/Databases/icsd.html)

BioMagResBank (www.bmrb.wisc.edu)

6 IUCr approved dictionaries are listed at www.iucr.org/iucr-top/cif/\#dics 
7 Hall, S.R.; Cook, A.P.F. STAR Data Definition Language: Initial Specification. J. Chem. Inform. Comp. Sci. 1995, 35, 819-825.

8 Westbrook, J.D.; Hall, S.R. A Dictionary Description Language for Macromolecular Structure. Draft DDL V 2.1.1 1995 (available from ndbserver.rutgers.edu/mmcif/ddl)

9 IUCr PDF verion of the 2.1 Core CIF Dictionary (available from ftp://ftp.iucr.org/cif_core_2.1.dic.pdf)

10 Acta Crystallographica validation criteria (www.iucr.org/iucr-top/journals/acta/dv.html)

11 Backus, J. Can Programming Be Liberated from the von Neumann Style? A Functional Style and Its Algebra of Programs. Communications ACM 1978, 21, 613-641

12 Halfant, M.; Sussman, G.J. Abstraction in Numerical Methods. Lisp and Functional Programming ACM 1988, 1-7

13 Hughes, J. Why Functional Programming Matters. Computer Journal 1989, 32, 98-107 


\section{CHART 1}

Example definitions from a prototype dictionary which includes method attributes

(identified by the tag_method.expression).

\section{a. Dictionary definition of crystal density.}

\begin{tabular}{|ll|}
\hline _definition.id & 'crystal.density' \\
_description.text & \\
; content. & \\
; Crystal density calculated from crystal unit cell and atomic \\
$\quad$ _type.container & Single \\
_type.value & Real \\
_enumeration.range & 0.0 : \\
$\quad$ _units.code & megagrams_per_metres_cubed \\
_method.expression & \\
; _crystal.density <- 1.6605 *_cell.mass /_cell.volume \\
\hline
\end{tabular}

b. Dictionary definition of cell volume.

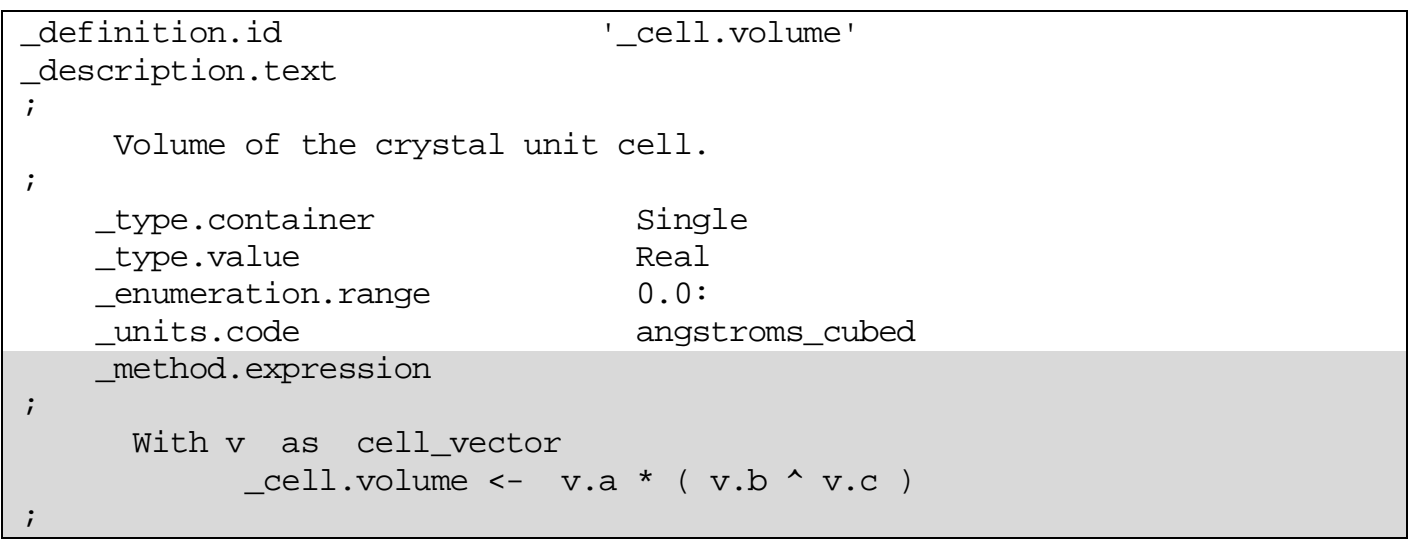

\section{c. Dictionary definition of cell mass.}

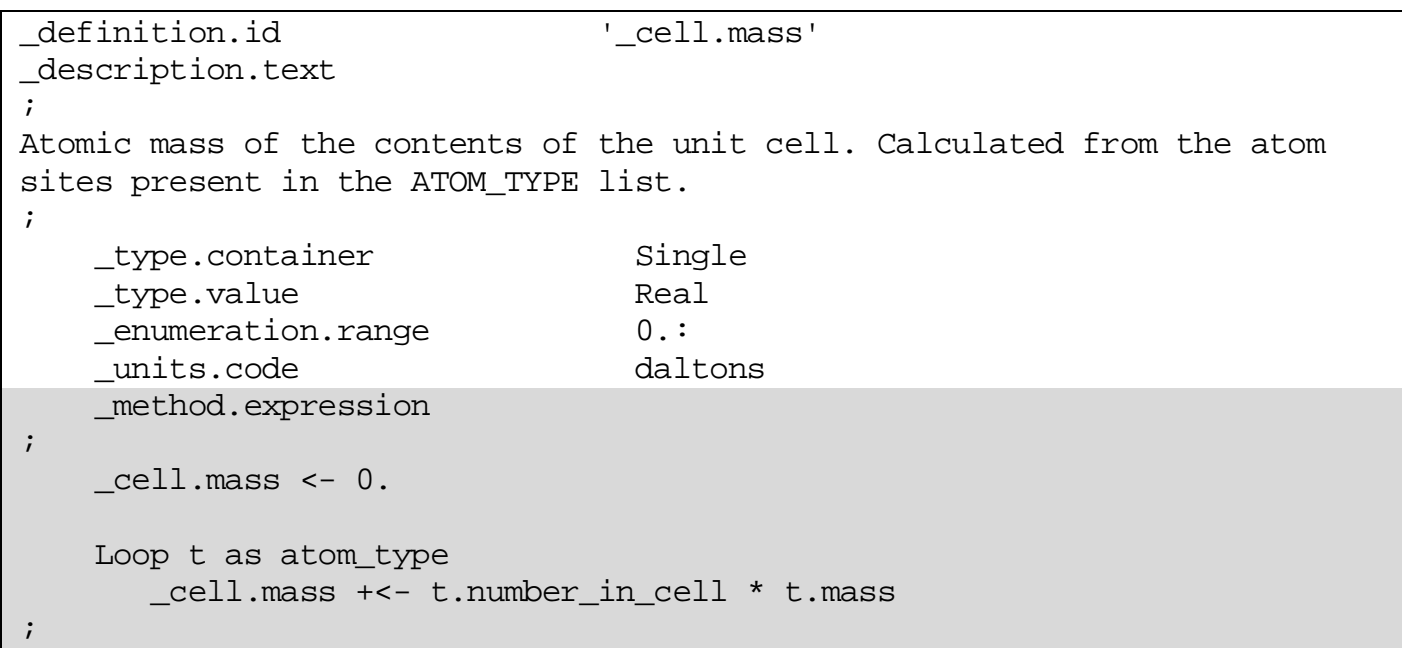




\section{CHART 2}

An example STAR data file that is applied in later example methods. This file contains a mixture of primitive and derivative data specifying the crystal structure of the molecule shown below. Note that there is a two-fold symmetry rotor at the centre of the ring, and the atom sites flagged with an asterisk are implied by this symmetry. The crystal density is included as an unknown value i.e. "?".

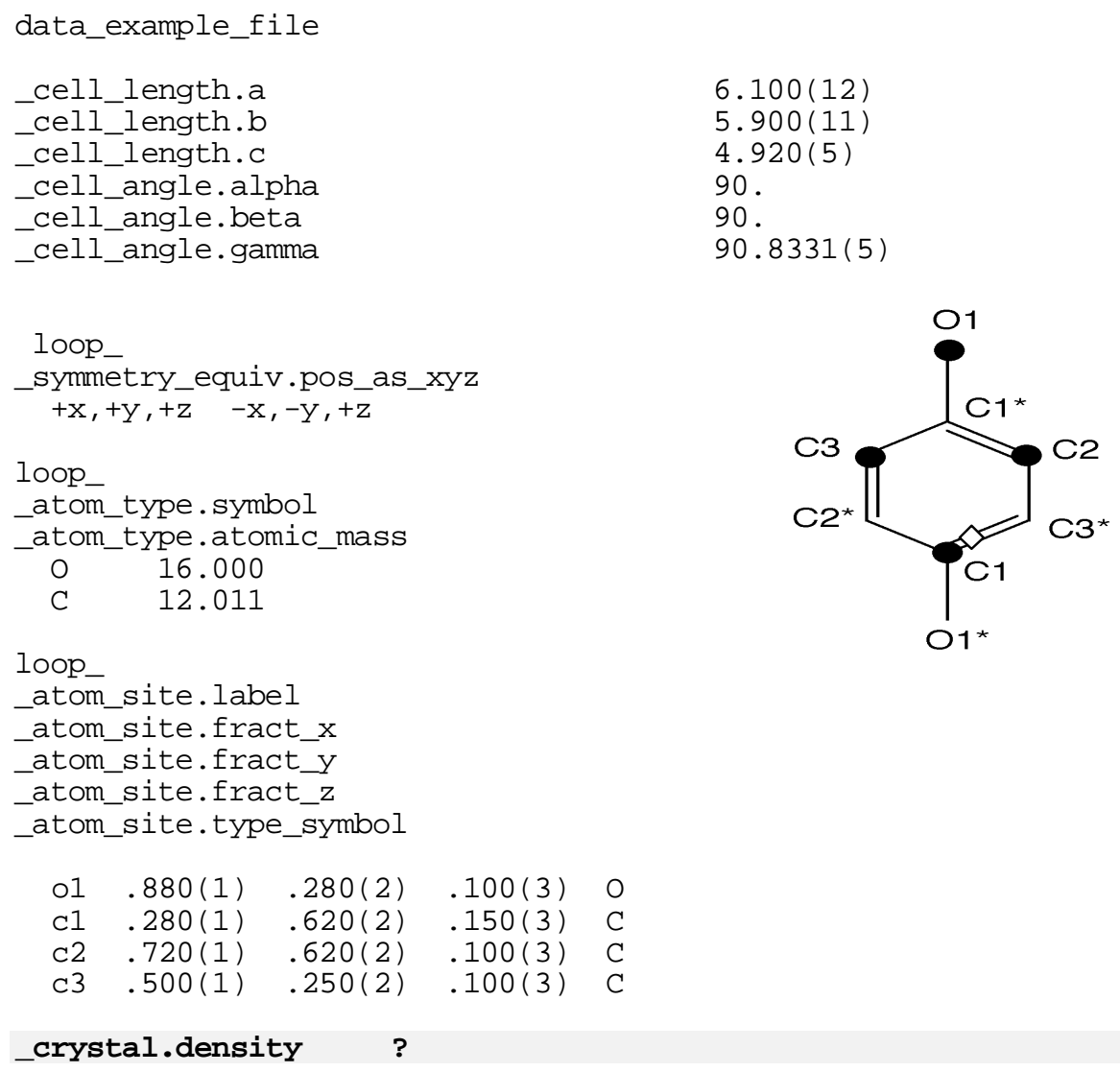




\section{CHART 3}

The output data file from a dREL run. The input file is shown in CHART 2. The data items determined from dictionary methods are in bold font.

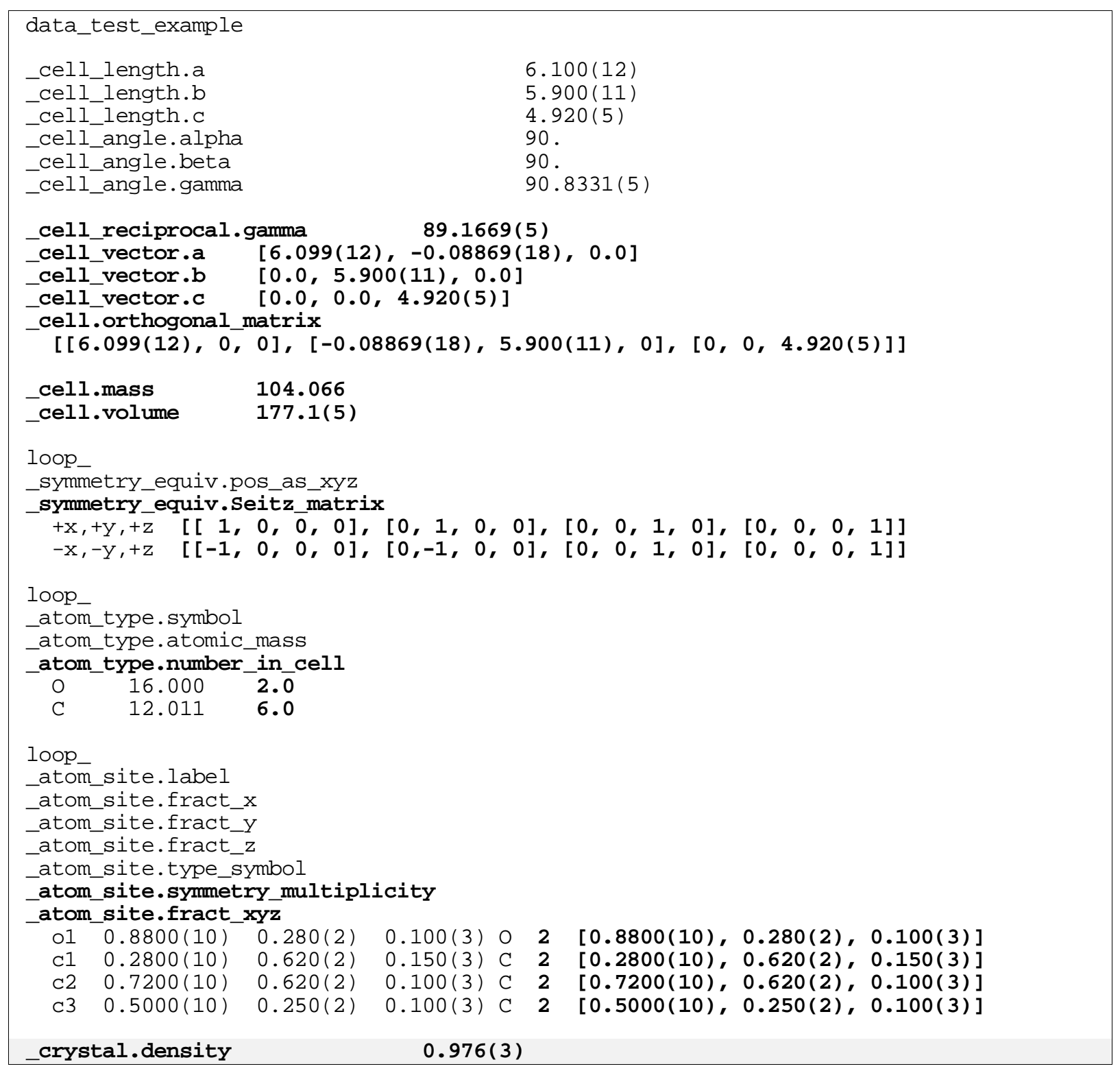




\section{CHART 4}

Definitions of hierarchically related categories, showing that the category key items for the ATOM_SITE_ANISO category is identically equivalent to the key for the parent category ATOM_SITE. See CHART 5 for an example of this sort of data.

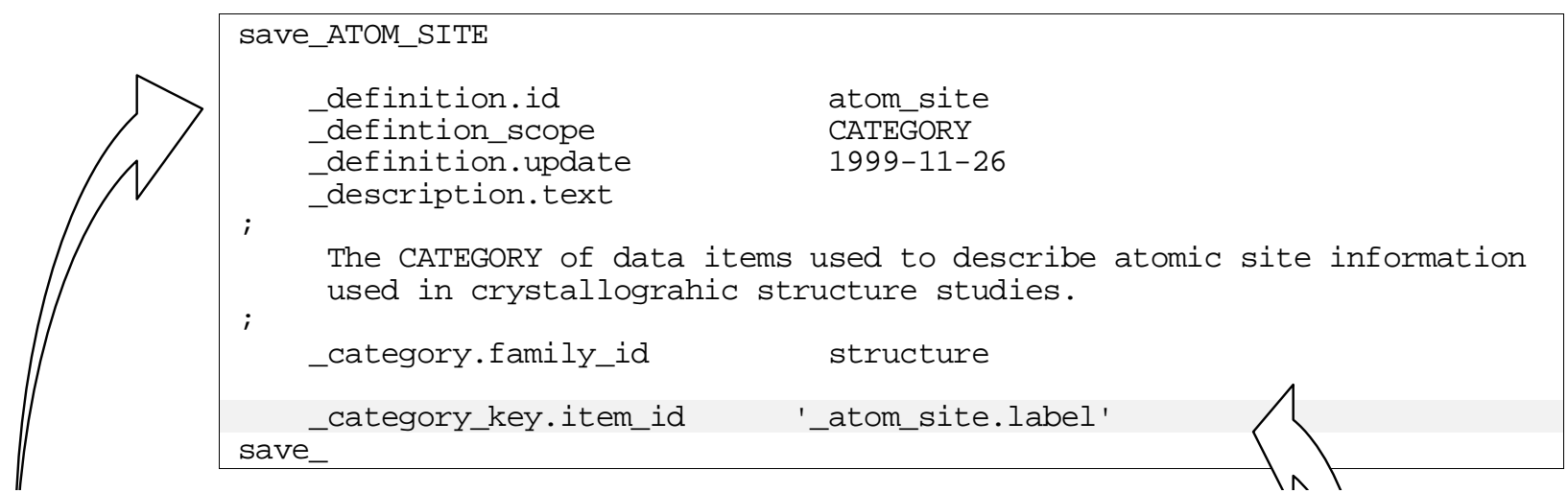

\section{Parent category}

Equivalent keys

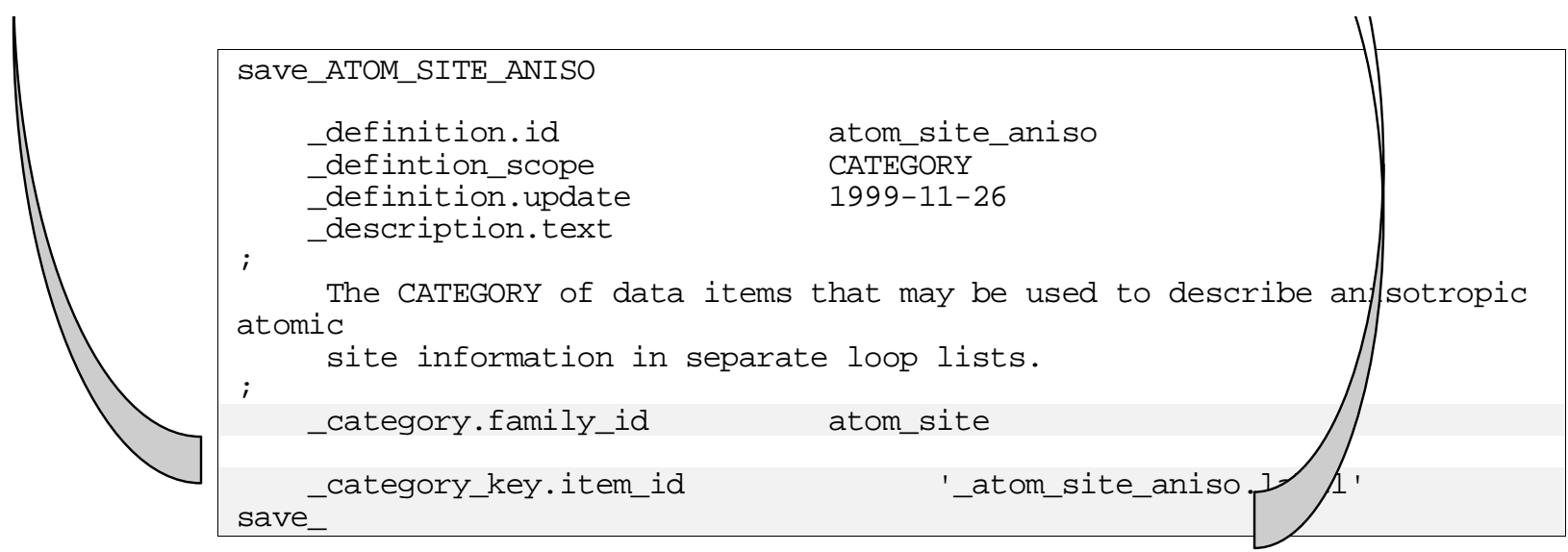




\section{CHART 5a}

Contents of a data file showing atom site data as separate list of coordinates and a list $U$ values. As shown in CHART 4, the highlighted items are category keys for referencing packets of items in these lists.

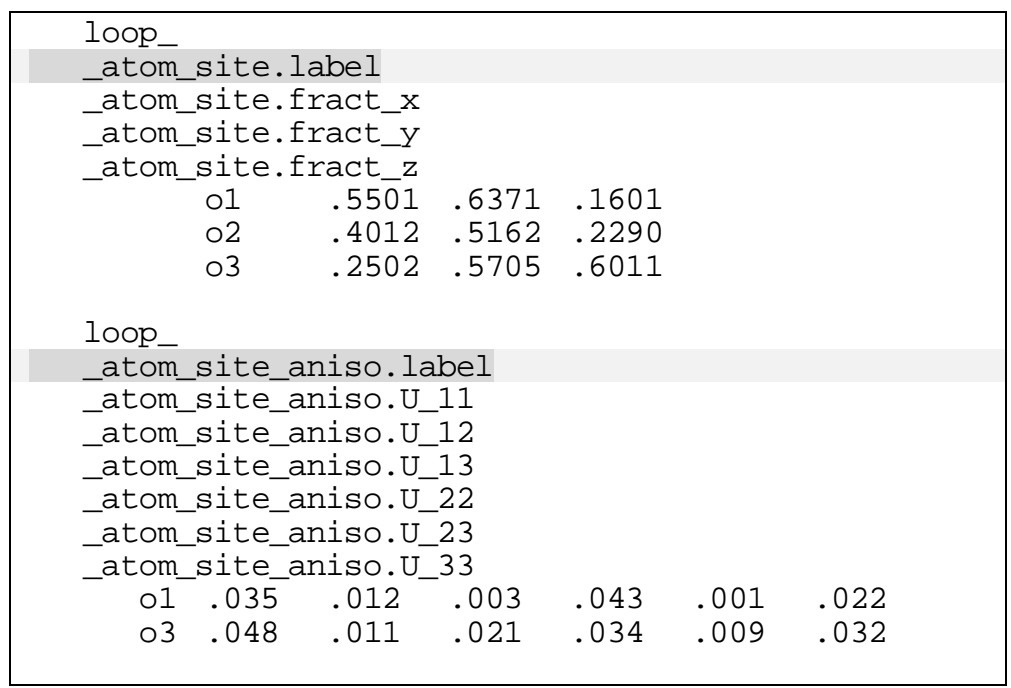

\section{CHART 5b}

This data file contains the identical atom site items as in CHART 5a but now as a single list. Because the category ATOM_SITE_ANISO is linked hierarchically to the category ATOM_SITE, the single reference key_atom_site. label may be used in a merged list.

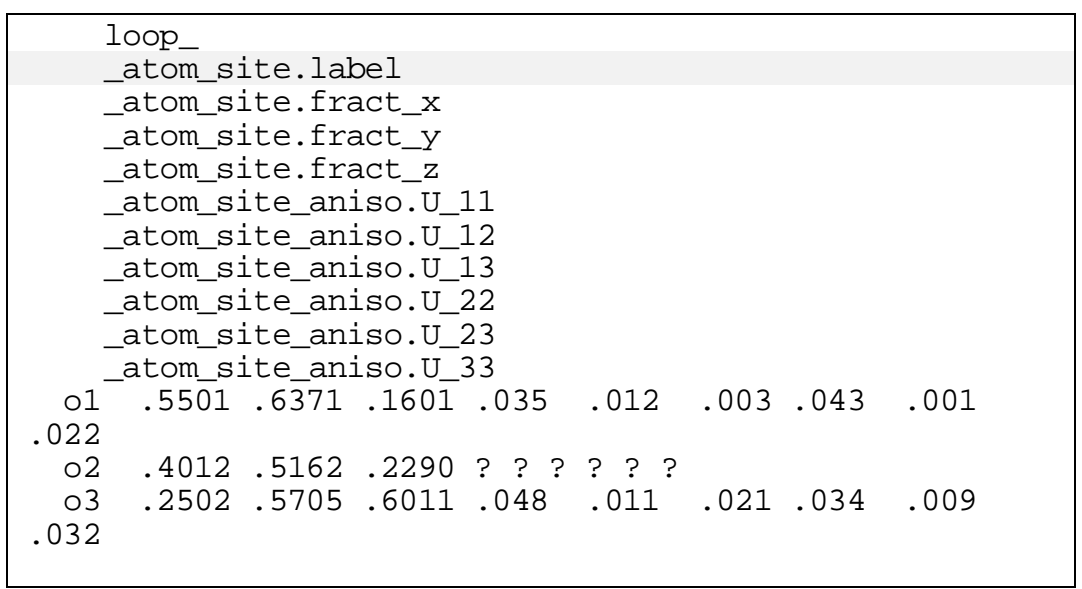




\section{CHART 6}

The dictionary definition of the complex structure factor.

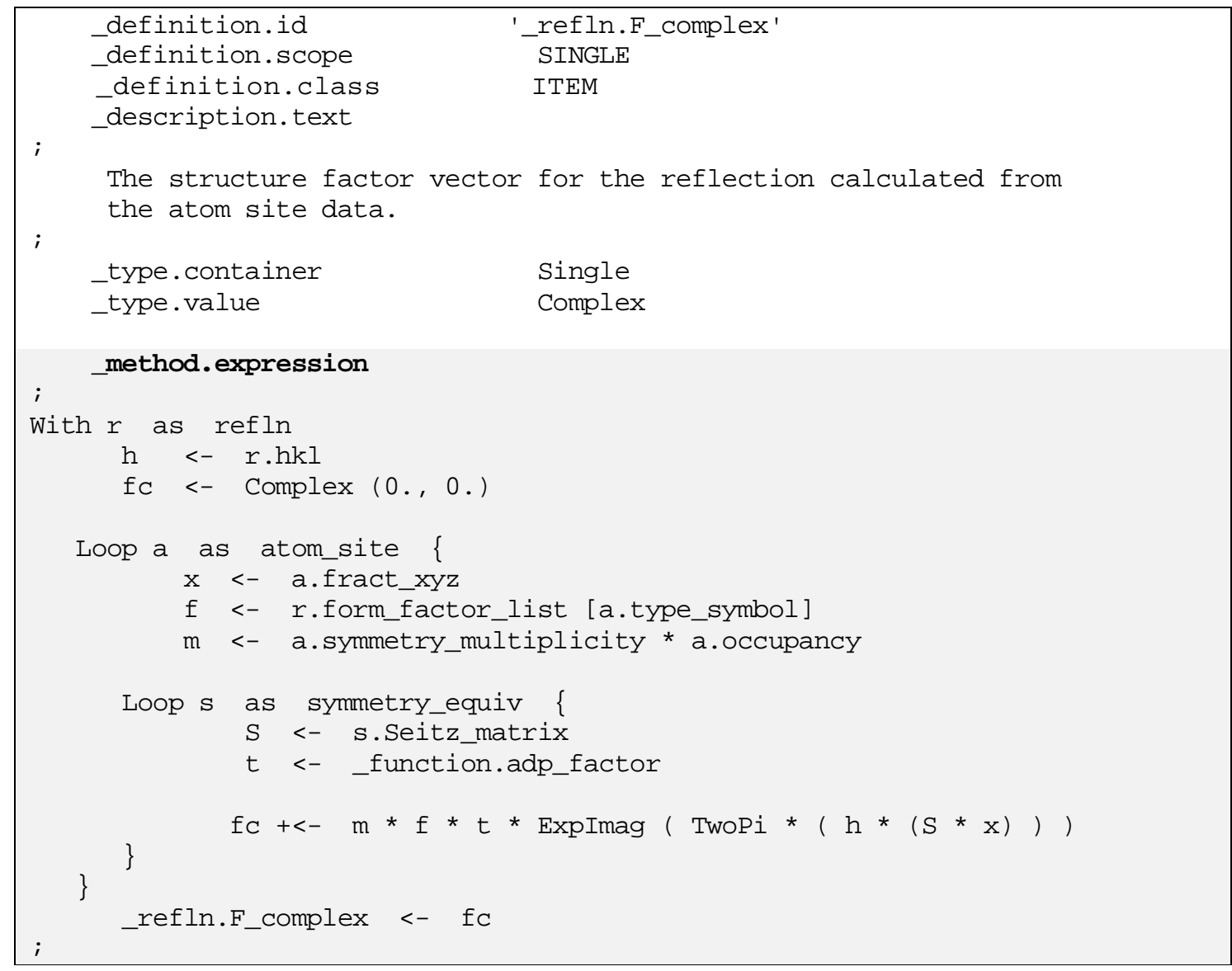




\section{CHART 7}

A typical derivation pathway for the evaluation of the structure factor.

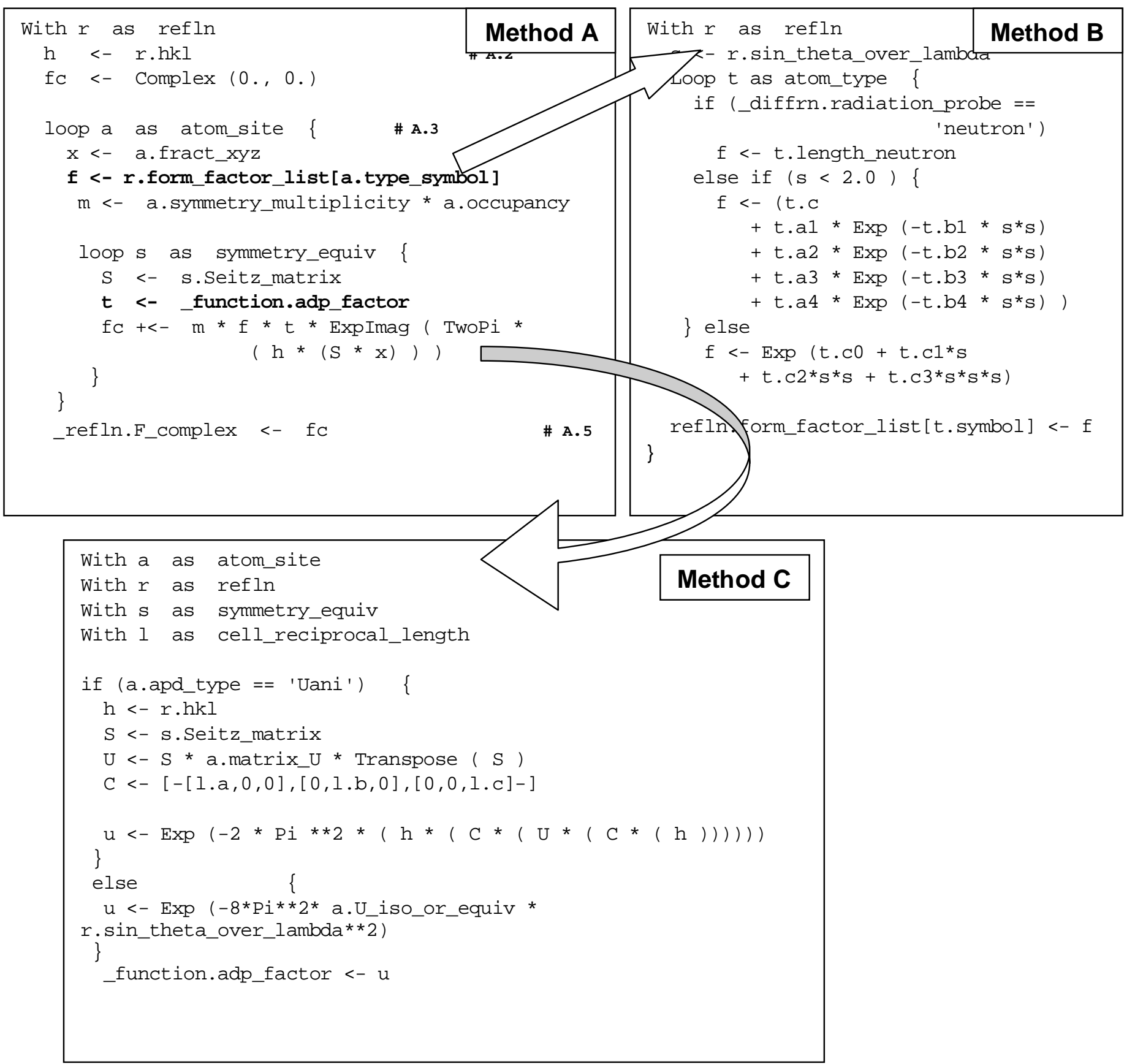




\section{CHART 8}

The definition of the data category GEOM_ANGLE showing the method attribute that generates a complete list of molecular geometry angles from the MODEL_SITE list.

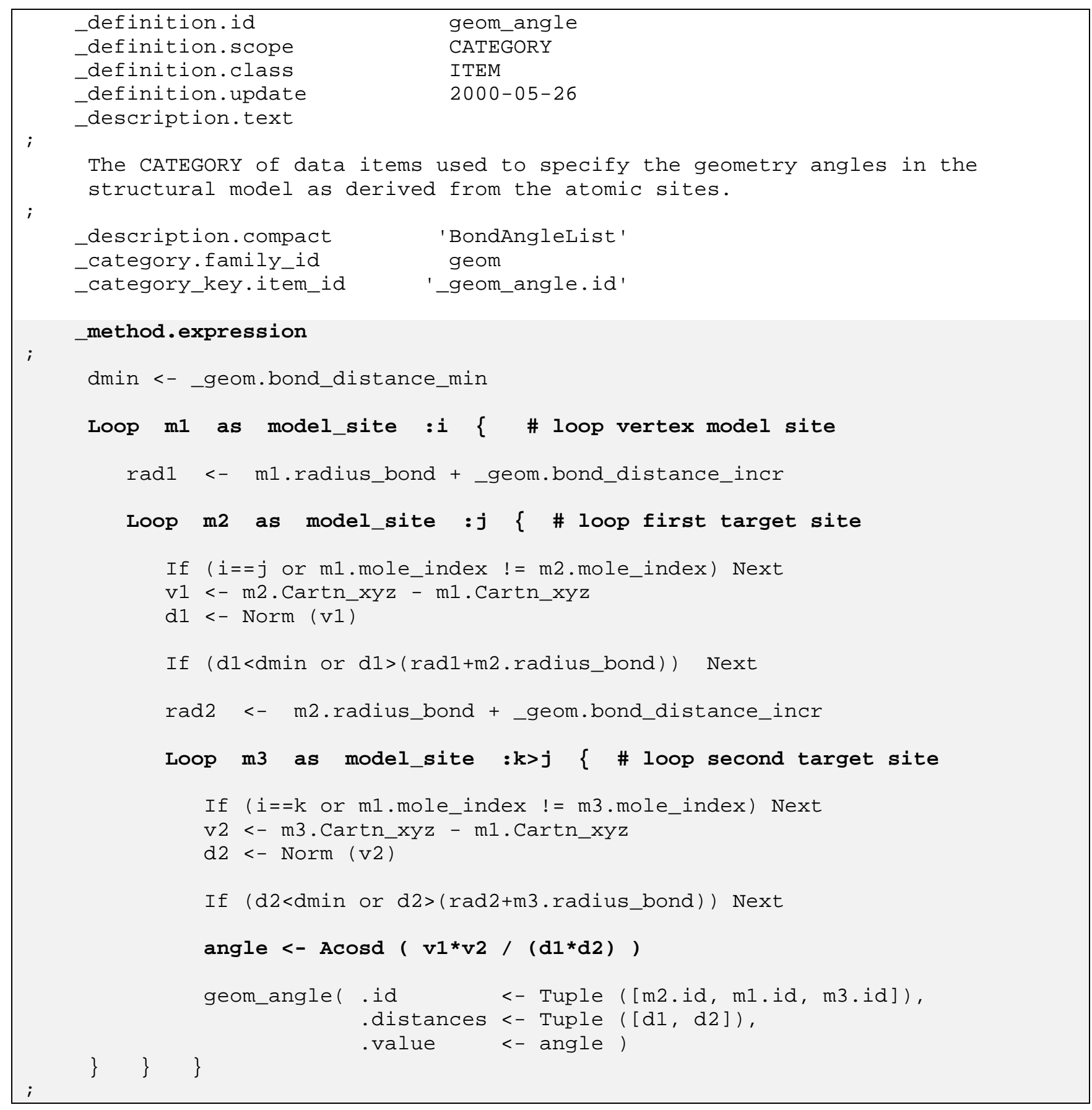




\section{CHART 9a}

An excerpt from a data file output from a $d R E L$ run showing the generated MODEL_SITE data.

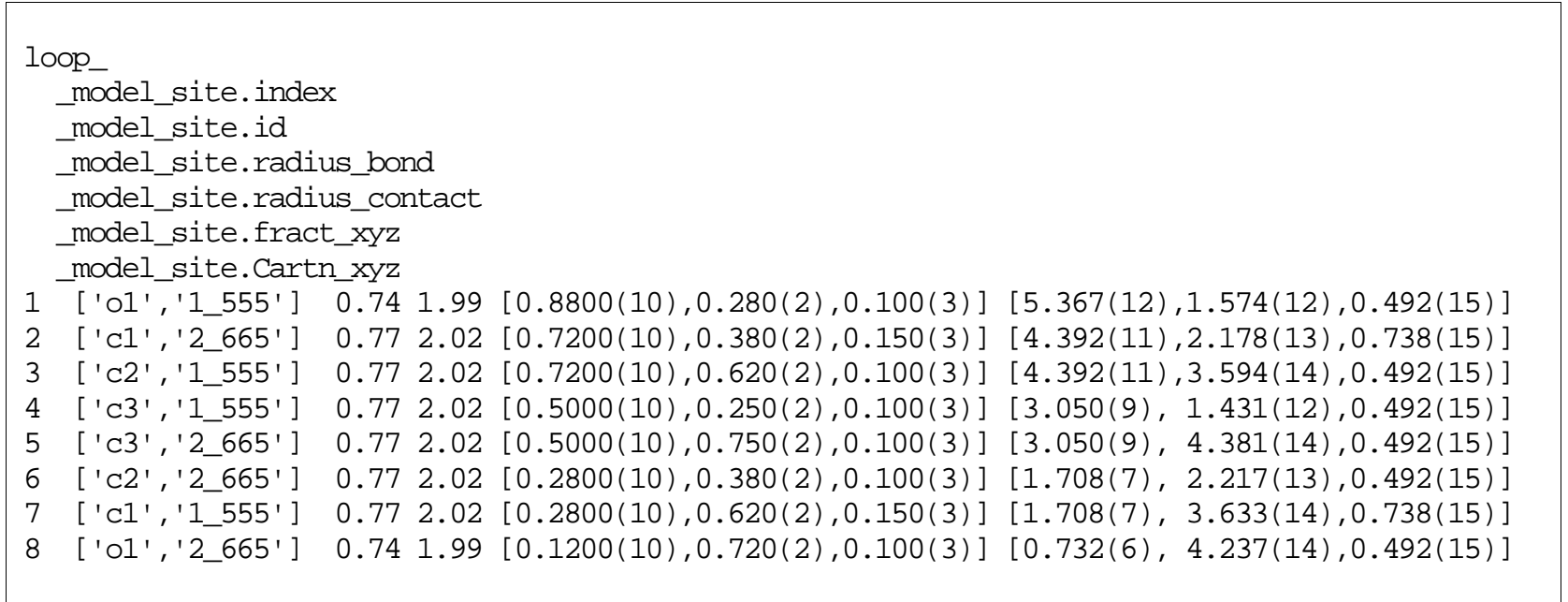

\section{CHART 9b}

An excerpt of a data file output from a $d R E L$ run showing the generated GEOM_ANGLE data.

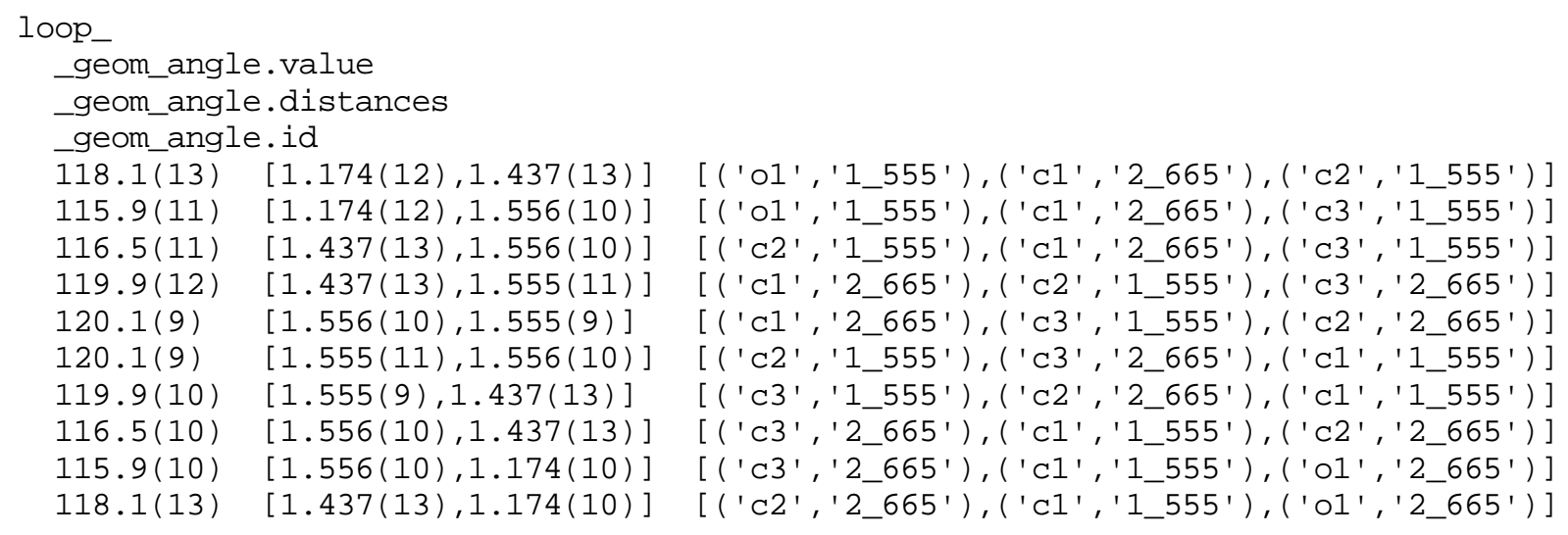

